\title{
ZIEBUR'S MATRIX EQUATION FOR POPULATION GROWTH
}

\author{
A. BROWN ${ }^{1}$
}

(Received 21 October 1985)

\begin{abstract}
The paper examines a matrix equation given by Ziebur [6] for the growth of a population in which the birth-rate and death-rate are age-dependent. For convenience the population was sub-divided into four age groups, with the same birth-rate and death-rate for individuals in a particular group, and the matrix equation relates the numbers in each sub-division in consecutive years. This avoids delay terms and makes it easier to modify the growth equation but it is shown that the form suggested by Ziebur for the transition matrix leads to some difficulties.
\end{abstract}

\section{Introduction}

In discussing population growth, mathematical models which allow for the age structure of the population have been used for some time [4, 5]. This approach was stimulated by two papers by Leslie $[2,3]$ who set up a matrix equation for the number of females in a population whose fertility rate and mortality rate varied with age. Lefkovitch [1] examined the practical problem of applying these ideas to data from laboratory experiments on a population of beetles where the age of each beetle could not be established precisely but different stages of development could be recognised. More recently, Ziebur [6] has shown how equations of this type can be modified to include other effects, such as the effect of growth to a limit and the effect of harvesting. To illustrate these ideas he considered a population which could be divided into four age classes, with uniform birth and death rates in each class, and put forward a matrix equation to relate the number in each class in consecutive years. The purpose of the present paper is to examine

\footnotetext{
${ }^{1}$ Department of Theoretical Physics, Research School of Physical Sciences, Australian National University, G.P.O. Box 4, Canberra, A.C.T. 2601

(C) Copyright Australian Mathematical Society 1986, Serial-fee code 0334-2700/86
} 
this basic equation and see what consequences it entails, since it involves an assumption about the distribution among the age groups within each of the four classes.

Section 2 specifies the model in more detail and introduces the notation that is employed. It also indicates which terms in the basic equation are of special interest in the later discussion. In section 3, a number of equations are established which are valid in the general case and in section 4 they are compared with the matrix equation proposed by Ziebur. It appears from this that Ziebur's equation imposes severe restrictions on the parameters and on the type of solution available.

\section{Notation and basic matrix equation}

To simplify comparison we shall follow Ziebur's model, although with some minor differences in notation. We can take the basic unit of time as a year in the discussion, and speak of population changes from one year to another, without worrying about the possibility that some other unit of time might be more appropriate for certain populations. Let

$$
x(j, n)=\text { number of individuals in class } j \text { in year } n,
$$

with $j=1,2,3,4$. Class 1 consists of individuals in the age group $\left(0, r_{1}\right)$, Class 2 corresponds to the age group $\left(r_{1}, r_{1}+r_{2}\right)$, class 3 to the age group $\left(r_{1}+r_{2}, r_{1}+\right.$ $\left.r_{2}+r_{3}\right)$ and Class 4 to the age group $\left(r_{1}+r_{2}+r_{3}, \infty\right)$. The annual birth and death rates are taken as uniform in each class, with $b$, and $d$, as the appropriate rates for Class $j$. Following Ziebur, we take $b_{j} \geqslant 0$ and $0<d_{j}<1$ for each $j$, so that the population fades away rather than dying at a fixed age. If $\mathbf{x}(n)$ denotes the vector $\{x(1, n), x(2, n), x(3, n), x(4, n)\}$, then Ziebur's basic equation is that

$$
\mathbf{x}(n+1)=L \mathbf{x}(n)
$$

where $L$ is a $4 \times 4$ matrix given by

$$
L=\left[\begin{array}{cccc}
\left(1-1 / r_{1}\right)\left(1-d_{1}\right)+b_{1} & b_{2} & b_{3} & b_{4} \\
\left(1-d_{1}\right) / r_{1} & \left(1-1 / r_{2}\right)\left(1-d_{2}\right) & 0 & 0 \\
0 & \left(1-d_{2}\right) / r_{2} & \left(1-1 / r_{3}\right)\left(1-d_{3}\right) & 0 \\
0 & 0 & \left(1-d_{3}\right) / r_{3} & 1-d_{4}
\end{array}\right]
$$

If we write

$$
B(n+1)=b_{1} x(1, n)+b_{2} x(2, n)+b_{3} x(3, n)+b_{4} x(4, n),
$$

then $B(n+1)$ is the number of individuals born during year $n$, which is the same as the number in the age group $(0,1)$ for year $n+1$. (It is assumed here that the census for year $n+1$ is taken at the beginning of the year, that is at the end of 
year $n$.) Thus we have

$$
x(1, n+1)=B(n+1)+\left(1-d_{1}\right) x(1, n)-\left(1 / r_{1}\right)\left(1-d_{1}\right) x(1, n)
$$

and we can interpret $\left(1-d_{1}\right) x(1, n)$ as the number of individuals from Class 1 who survive to the end of year $n$, while the last term represents the number transferred from Class 1 to Class 2 at the end of year $n$. This implies that the number in the age group $\left(r_{1}-1, r_{1}\right)$ at the beginning of year $n$ was $\left(1 / r_{1}\right) x(1, n)$, with the survivors counted in age group $\left(r_{1}, r_{1}+1\right)$ in the census for year $n+1$. There is no difficulty about this if $r_{1}=1$ but if $r_{1}=2$ we immediately have equal numbers in age groups $(0,1)$ and $(1,2)$ at the beginning of year $n$. [We have $(1 / 2) x(1, n)$ in age group $(1,2)$, so we must have $(1 / 2) x(1, n)$ in age group $(0,1)$.] This holds for all values of $n$, which means that we have a restriction on the population. Indeed it can be deduced that $B(n+1)<B(n)$, which is a strong restriction, but we shall return to this later.

What happens for $r_{1} \geqslant 3$ ? Are the individuals in Class 1 uniformly distributed over the age groups $(0,1),(1,2), \ldots,\left(r_{1}-1, r_{1}\right)$ ? Perhaps not, since $\left(1 / r_{1}\right) x(1, n)$ is only an average over the different age groups for year $n$, so it would be possible to have this number in age group $\left(r_{1}-1, r_{1}\right)$ without having a uniform distribution. Yet this formula has to hold for all values of $n$ and we can anticipate that this will bring in relationships between the numbers in different one-year intervals.

A similar problem arises with the number of individuals transferred from Class 2 to Class 3 and from Class 3 to Class 4 at the end of year $n$. These numbers are

$$
\left(1 / r_{2}\right)\left(1-d_{2}\right) x(2, n) \text { and }\left(1 / r_{3}\right)\left(1-d_{3}\right) x(3, n) \text {, }
$$

respectively, as can be seen from equations (2.2) and (2.3). This means that at the beginning of year $n$ there were

(i) $\left(1 / r_{2}\right) x(2, n)$ individuals in the age group $\left(r_{1}+r_{2}-1, r_{1}+r_{2}\right)$,

(ii) $\left(1 / r_{3}\right) \times(3, n)$ individuals in the age group $\left(r_{1}+r_{2}+r_{3}-1, r_{1}+r_{2}+r_{3}\right)$. We can guess that these expressions will raise questions similar to those that came up in the previous paragraph.

\section{Equations relating $x(j, n)$ to $B(n)$}

To look at these problems in a different way, let us start with $B(n)$ individuals in the age group $(0,1)$ in year $n$. This produces

$$
\begin{aligned}
& \left(1-d_{1}\right) B(n) \text { in age group }(1,2) \text { in year } n+1 \\
& \left(1-d_{1}\right)^{2} B(n) \text { in age group }(2,3) \text { in year } n+2 \\
& \ldots \ldots \ldots \ldots \ldots \ldots \ldots \ldots \ldots \ldots \ldots \ldots \ldots \ldots \ldots \ldots \ldots \ldots \ldots \\
& \left(1-d_{1}\right)^{r_{1}-1} B(n) \text { in age group }\left(r_{1}-1, r_{1}\right) \text { in year } n+r_{1}-1 .
\end{aligned}
$$


It follows that (for $n=0,1,2, \ldots$ )

$$
\begin{aligned}
x\left(1, n+r_{1}-1\right)= & \left(1-d_{1}\right)^{r_{1}-1} B(n) \\
& +\left(1-d_{1}\right)^{r_{1}-2} B(n+1)+\cdots B\left(n+r_{1}-1\right) \\
= & \sum_{k=n}^{n+r_{1}-1} B(k)\left(1-d_{1}\right)^{n+r_{1}-k-1} .
\end{aligned}
$$

If we extend the argument above we get

$$
\begin{aligned}
& \left(1-d_{1}\right)^{r_{1}} B(n) \text { in age group }\left(r_{1}, r_{1}+1\right) \text { in year } n+r_{1} \\
& \left(1-d_{2}\right)\left(1-d_{1}\right)^{r_{1}} B(n) \text { in age group }\left(r_{1}+1, r_{1}+2\right) \text { in year } n+r_{1}+1 ; \\
& \left(1-d_{2}\right)^{r_{2}-1}\left(1-d_{1}\right)^{r_{1}} B(n) \text { in age group }\left(r_{1}+r_{2}-1, r_{1}+r_{2}\right) \text { in year } \\
& n+r_{1}+r_{2}-1 ;
\end{aligned}
$$

and consequently

$$
x\left(2, n+r_{1}+r_{2}-1\right)=\left(1-d_{1}\right)^{r_{1}} \sum_{k=n}^{n+r_{2}-1} B(k)\left(1-d_{2}\right)^{n+r_{2}-k-1} .
$$

A similar extension of the argument gives

$x\left(3, n+r_{1}+r_{2}+r_{3}-1\right)=\left(1-d_{1}\right)^{r_{1}}\left(1-d_{2}\right)^{r_{2}} \sum_{k=n}^{n+r_{3}-1} B(k)\left(1-d_{3}\right)^{n+r_{3}-k-1}$.

For $x(4, n)$ we have to count over an infinite sequence of one-year intervals and this puts a strain on the notation. We can get round this by postulating that in year zero there were

$B(0)$ individuals in the age group $(0,1)$;

$\left(1-d_{1}\right) B(-1)$ individuals in the age group $(1,2)$;

$\left(1-d_{1}\right)^{2} B(-2)$ individuals in the age group $(2,3)$;

$\left(1-d_{1}\right)^{r_{1}} B\left(-r_{1}\right)$ individuals in the age group $\left(r_{1}, r_{1}+1\right)$;

$\left(1-d_{2}\right)\left(1-d_{1}\right)^{r_{1}} B\left(-r_{1}-1\right)$ individuals in the age group $\left(r_{1}+1, r_{1}+2\right)$;

$\left(1-d_{3}\right)\left(1-d_{2}\right)^{r_{2}}\left(1-d_{1}\right)^{r_{1}} B\left(-r_{1}-r_{2}-1\right)$ individuals in the age group

$\left(r_{1}+r_{2}+1, r_{1}+r_{2}+2\right)$;

and so on. This defines $B(-n)$ and allows us to write $x\left(4, n+r_{1}+r_{2}+r_{3}\right)$

$$
=\left(1-d_{1}\right)^{r_{1}}\left(1-d_{2}\right)^{r_{2}}\left(1-d_{3}\right)^{r_{3}} \sum_{k=-\infty}^{n} B(k)\left(1-d_{4}\right)^{n-k} .
$$


From equation (3.1)

$$
\begin{aligned}
x\left(1, n+r_{1}+1\right)-\left(1-d_{1}\right) x\left(1, n+r_{1}\right) & \\
= & \left(\sum_{k=n+2}^{n+r_{1}+1}-\sum_{k=n+1}^{n+r_{1}}\right)\left\{B(k)\left(1-d_{1}\right)^{n+r_{1}+1-k}\right\} \\
= & B\left(n+r_{1}+1\right)-\left(1-d_{1}\right)^{r_{1}} B(n+1) .
\end{aligned}
$$

Similarly, it can be shown that

$$
\begin{gathered}
x\left(2, n+r_{1}+r_{2}+1\right)-\left(1-d_{2}\right) x\left(2, n+r_{1}+r_{2}\right) \\
=\left(1-d_{1}\right)^{r_{1}}\left\{B\left(n+r_{2}+1\right)-\left(1-d_{2}\right)^{r_{2}} B(n+1)\right\} \\
x\left(3, n+r_{1}+r_{2}+r_{3}+1\right)-\left(1-d_{3}\right) x\left(3, n+r_{1}+r_{2}+r_{3}\right) \\
=\left(1-d_{1}\right)^{r_{1}}\left(1-d_{2}\right)^{r_{2}}\left\{B\left(n+r_{3}+1\right)-\left(1-d_{3}\right)^{r_{3}} B(n+1)\right\} \\
x\left(4, n+r_{1}+r_{2}+r_{3}+1\right)-\left(1-d_{4}\right) x\left(4, n+r_{1}+r_{2}+r_{3}\right) \\
=\left(1-d_{1}\right)^{r_{1}}\left(1-d_{2}\right)^{r_{2}}\left(1-d_{3}\right)^{r_{3}} B(n+1) .
\end{gathered}
$$

If we write

$$
\begin{aligned}
& P(n)=\sum_{j=1}^{4} x(j, n)=\text { total population in year } n, \\
& D(n)=\sum_{j=1}^{4} d_{j} x(j, n)=\text { number of deaths in year } n,
\end{aligned}
$$

equations (3.5), (3.6), (3.7) and (3.8) can be used together to give

$$
\begin{aligned}
P(n & \left.+r_{1}+r_{2}+r_{3}+1\right)-P\left(n+r_{1}+r_{2}+r_{3}\right) \\
& =B\left(n+r_{1}+r_{2}+r_{3}+1\right)-D\left(n+r_{1}+r_{2}+r_{3}\right)
\end{aligned}
$$

or, replacing $n+r_{1}+r_{2}+r_{3}$ by $m$,

$$
P(m+1)=P(m)+B(m+1)-D(m) .
$$

This serves as a check since it gives the obvious result that the increase in population size from year $m$ to year $m+1$ equals the excess of births over deaths in year $m$.

\section{Effect of Ziebur's form for $L$}

The equations in section 3 show that the $x(j, n)$ can be related to the sequence $\{B(n)\}$ and it should be noted that these equations do not depend on the form for $L$. However, using equation (2.3) introduces additional constraints on $\{B(n)\}$ and it is these additional constraints that we want to examine. We saw that if we 
start with $B(n)$ individuals in the age group $(0,1)$ in year $n$ there will be $\left(1-d_{1}\right)^{r_{1}} B(n)$ individuals in the age group $\left(r_{1}, r_{1}+1\right)$ in year $n+r_{1}$. These are the individuals transferred from Class 1 to Class 2 at the end of year $n+r_{1}-1$, so with Ziebur's form for $L$ we must have

$$
\left(1-d_{1}\right)^{r_{1}} B(n)=\left(1 / r_{1}\right)\left(1-d_{1}\right) x\left(1, n+r_{1}-1\right),
$$

that is

$$
x\left(1, n+r_{1}-1\right)=r_{1}\left(1-d_{1}\right)^{r_{1}-1} B(n) .
$$

As noted before, there is no problem when $r_{1}=1$ for in this case $x(1, n)=B(n)$ and there is only one year group involved. (This could be appropriate, for example, in populations where the mortality rate is exceptionally high in the first year and has to be considered separately.) For $r_{1}=2$, equations (4.2) and (3.1) give

$$
x(1, n+1)=2\left(1-d_{1}\right) B(n)=B(n+1)+\left(1-d_{1}\right) B(n)
$$

and hence

$$
B(n+1)=\left(1-d_{1}\right) B(n) .
$$

This checks with an earlier comment that the number of individuals in age group $(0,1)$, namely $B(n+1)$, is equal to the number in age group $(1,2)$, which is $\left(1-d_{1}\right) B(n)$. Also, equation (4.3) gives $B(n)=B(0)\left(1-d_{1}\right)^{n}$ as its solution. Since $0<1-d_{1}<1$, we see that $B(n+1)<B(n)$ and $B(n)$ decreases exponentially as $n$ increases.

For $r_{1} \geqslant 3$, we can combine equation (4.2) with equation (3.1) to obtain

$$
r_{1}\left(1-d_{1}\right)^{r_{1}-1} B(n)=\sum_{k=n}^{n+r_{1}-1} B(k)\left(1-d_{1}\right)^{n+r_{1}-k-1},
$$

which is a linear equation for $B(n)$, with constant coefficients. If we look for a solution of the form $B(n)=A \rho^{n}$, with $\rho \neq 0$, then $\rho$ must be a root of the polynomial equation

$$
r_{1}=\sum_{m=0}^{r_{1}-1} \rho^{m}\left(1-d_{1}\right)^{-m}=\sum_{m=0}^{r_{1}-1} \sigma_{1}^{m},
$$

where $\sigma_{1}=\rho /\left(1-d_{1}\right)$. Clearly, $\sigma_{1}=1$ is a solution of this equation, with $\rho=1-d_{1}$ as the corresponding value of $\rho$. Thus we again get $B(n)=$ $\left(1-d_{1}\right)^{n} B(0)$ as a possible solution and this gives a situation where $x(1, n)$ is divided equally among the age groups $(0,1),(1,2), \ldots,\left(r_{1}-1, r_{1}\right)$, with $B(n)$ decreasing exponentially as $n$ increases. In this case $x(1, n)=r_{1} B(n)$, so $x(1, n)$ also decreases exponentially as $n$ increases.

The remaining roots of equation (4.5) come from $G\left(\sigma_{1}, r_{1}\right)=0$, where

$$
G\left(\sigma_{1}, r_{1}\right)=\sum_{m=1}^{r_{1}-1} m \sigma_{1}^{r_{1}-m-1},
$$


and we can say right away that this equation has no positive roots. It can also be shown that there are no roots with modulus less than 1 . To see this, consider the right-hand side of equation (4.5) when $\left|\sigma_{1}\right|=R$, with $0 \leqslant R<1$. Then

$$
\left|1+\sigma_{1}+\sigma_{1}^{2}+\cdots+\sigma_{1}^{r_{1}-1}\right| \leqslant 1+R+R^{2}+\cdots+R^{r_{1}-1}<r_{1},
$$

so equation (4.5) has no roots with $\left|\sigma_{1}\right|<1$. When $\left|\sigma_{1}\right|=R$, with $R=1$,

$$
\left|1+\sigma_{1}+\sigma_{1}^{2}+\cdots+\sigma_{1}^{r_{1}-1}\right| \leqslant 1+R+R^{2}+\cdots+R^{r_{1}-1}=r_{1},
$$

but the equality only holds when $\sigma_{1}=1$, which is not a root of $G\left(\sigma_{1}, r_{1}\right)=0$. Thus $\left|\sigma_{1}\right|>1$ for each root of $G\left(\sigma_{1}, r_{1}\right)=0$.

This means that $\rho=1-d_{1}$ is the solution for $\rho$ with the smallest modulus, so if we write down the general solution for $B(n)$ from equation (4.4) the term involving $\left(1-d_{1}\right)^{n}$ will not be the dominant term (in general) for large values of $n$. It would be possible to choose the initial conditions so that $B(n)=A\left(1-d_{1}\right)^{n}$ is the only term in the solution but any disturbance or perturbation would bring in other terms which eventually dominate the solution. The dominant term would then correspond to a real value of $\sigma_{1}$, with $\sigma_{1}<-1$, or to a pair of complex conjugate values of $\sigma_{1}$ and in either case $B(n)$ would have negative values for some $n$, which does not make sense in the biological problem.

Similar difficulties arise if we consider the number of individuals transferred from Class 2 to Class 3 . If we start with $B(n)$ individuals in the age group $(0,1)$ in year $n$, then there will be $\left(1-d_{1}\right)^{r_{1}}\left(1-d_{2}\right)^{r_{2}} B(n)$ individuals in the age group $\left(r_{1}+r_{2}, r_{1}+r_{2}+1\right)$ in year $n+r_{1}+r_{2}$ and these are the individuals transferred from Class 2 to Class 3 at the end of year $n+r_{1}+r_{2}-1$. With Ziebur's form for $L$ the number transferred is $\left(1 / r_{2}\right)\left(1-d_{2}\right) x\left(2, n+r_{1}+r_{2}-1\right)$, so we must have

$$
r_{2}\left(1-d_{1}\right)^{r_{1}}\left(1-d_{2}\right)^{r_{2}-1} B(n)=x\left(2, n+r_{1}+r_{2}-1\right) \text {. }
$$

As before, there is no problem if $r_{2}=1$ and if $r_{2}=2$ there must be equal numbers in age groups $\left(r_{1}, r_{1}+1\right)$ and $\left(r_{1}+1, r_{1}+2\right)$, with $B(n+1)=$ $\left(1-d_{2}\right) B(n)$. We note that this equation is not consistent with $B(n+1)=$ $\left(1-d_{1}\right) B(n)$ unless $d_{1}=d_{2}$. For $r_{2} \geqslant 3$ we can combine equations (3.2) and (4.7) to give

$$
r_{2}\left(1-d_{1}\right)^{r_{1}}\left(1-d_{2}\right)^{r_{2}-1} B(n)=\left(1-d_{1}\right)^{r_{1}} \sum_{k=n}^{n+r_{2}-1} B(k)\left(1-d_{2}\right)^{n+r_{2}-k-1}
$$

or

$$
r_{2}\left(1-d_{2}\right)^{r_{2}-1} B(n)=\sum_{k=n}^{n+r_{2}-1} B(k)\left(1-d_{2}\right)^{n+r_{2}-k-1} .
$$

This again gives a linear equation for $B(n)$, with constant coefficients, and indeed equation (4.8) is identical with equation (4.4) except that $r_{2}$ replaces $r_{1}$ and $d_{2}$ replaces $d_{1}$. If $r_{2}=r_{1}$ and $d_{2}=d_{1}$ we get the same equation as before but in general the two equations will be incompatible. 
We can discuss the transfer of individuals from Class 3 to Class 4 in the same way. For $r_{3} \geqslant 3$, Ziebur's form for $L$ leads to the equation

$$
r_{3}\left(1-d_{3}\right)^{r_{3}-1} B(n)=\sum_{k=n}^{n+r_{3}-1} B(k)\left(1-d_{3}\right)^{n+r_{3}-k-1}
$$

and this is also identical with equation (4.4) except that $r_{3}$ replaces $r_{1}$ and $d_{3}$ replaces $d_{1}$. We can look for a solution for equation (4.9) in the same way as before but in general equations (4.4), (4.8) and (4.9) will be incompatible. The obvious exception is when $d_{1}=d_{2}=d_{3}$ and $r_{1}=r_{2}=r_{3}$ but this places a severe restriction on the parameters of the problem.

\section{Conclusion}

Using equations (2.2) and (2.3) as the basic relations for population growth offers a number of advantages and Ziebur exploits them with ingenuity in the later developments in his paper, where he modifies the equations, gives explicit forms of solution and discusses the behaviour of the solution for large values of $n$. $\mathrm{He}$ also uses $L$ in going from the difference equation to a corresponding differential equation and in turn modifying the differential equation. However, section 4 shows that his form for $L$ entails a number of difficulties. Equations (3.5) to (3.8) could be used instead, with $B(n+1)$ expressed in terms of $\mathbf{x}(n)$ from equation (2.4), but these equations involve delay terms and are less convenient to use. For example, equation (3.5) can be written as

$$
\begin{aligned}
& \text { Increase in Class } 1 \text { from year } n+r_{1} \text { to year } n+r_{1}+1 \\
&= x\left(1, n+r_{1}+1\right)-x\left(1, n+r_{1}\right) \\
&= B\left(n+r_{1}+1\right)-d_{1} x\left(1, n+r_{1}\right)-\left(1-d_{1}\right)^{r_{1}} B(n+1) \\
&=\left(\text { births in year } n+r_{1}\right)-(\text { deaths in Class } 1 \text { in year } \\
&\left.n+r_{1}\right)-(\text { number transferred to Class } 2 \text { at end of } \\
&\text { year } \left.n+r_{1}\right)
\end{aligned}
$$

and the last of these is the delay term since it involves $\mathrm{x}(n)$, the population vector $r_{1}$ years earlier.

Similarly, equation (3.6) can be thought of as

Increase in Class 2 from year $n+r_{1}+r_{2}$ to year $n+r_{1}+r_{2}+1$

$=$ (number transferred from Class 1$)-($ number transferred to Class 3) - (deaths in Class 2). 
Here there are two delay terms on the right-hand side, since the first transfer term involves $\mathbf{x}\left(n+r_{2}\right)$, a delay of $r_{1}$ years, and the second transfer term involves $\mathbf{x}(n)$, a delay of $r_{1}+r_{2}$ years. Equation (3.7) also involves two transfer terms, with a delay of $r_{1}+r_{2}$ years in one case and $r_{1}+r_{2}+r_{3}$ years in the other case, while equation (3.8) has a single delay term, with a delay of $r_{1}+r_{2}+r_{3}$ years. Ziebur avoids these delay terms by using

(i) $\left(1 / r_{1}\right) x\left(1, n+r_{1}\right)$ instead of $\left(1-d_{1}\right)^{r_{1}-1} B(n+1)$,

(ii) $\left(1 / r_{2}\right) \times\left(2, n+r_{1}+r_{2}\right)$ instead of $\left(1-d_{1}\right)^{r_{1}}\left(1-d_{2}\right)^{r_{2}-1} B(n+1)$,

(iii) $\left(1 / r_{3}\right) \times\left(3, n+r_{1}+r_{2}+r_{3}\right)$ instead of $\left(1-d_{1}\right)^{r_{1}}\left(1-d_{2}\right)^{r_{2}}$ $\cdot\left(1-d_{3}\right)^{r_{3}-1} B(n+1)$.

Although the appearance of the delay terms is logical, Ziebur's paper shows that there would be considerable advantages if the delay terms could be replaced by a suitable approximation to give a growth equation of the same form as equation (2.2). The approximation suggested by Ziebur produces difficulties but possibly an alternative can be found.

\section{Acknowledgment}

This work was carried out as a Visiting Fellow in the Research School of Physical Sciences, Australian National University, and I am grateful to the Department of Theoretical Physics for the facilities it has provided.

\section{References}

[1] L P. Lefkovitch, "The study of population growth in organisms grouped by stages", Biometrics 21 (1965), 1-18.

[2] P H. Leslie, "On the use of matrices in certain population mathematics", Biometrika 33 (1945), $183-212$.

[3] P. H. Leslie, "Some further notes on the use of matrices in population mathematics", Biometrika 35 (1948), 213-245.

[4] E. C. Pielou, An introduction to mathematical ecology (Wiley, New York, 1969), 33-42.

[5] M. H. Williamson, The analysis of biological populations (Arnold, London, 1972), 21-26.

[6] A. D. Ziebur, "Age-dependent models of population growth", Theoret. Population Biol. 26 (1984), 315-319. 